\title{
Ischemia Cerebrovascular, CTCAE
}

National Cancer Institute

\section{Source}

National Cancer Institute. Ischemia Cerebrovascular, CT CAE. NCI Thesaurus. Code C143619.

A disorder characterized by a decrease or absence of blood supply to the brain caused by obstruction (thrombosis or embolism) of an artery resulting in neurological damage. 